\title{
Arquitectura Escrita 2017
}

Con el objetivo de reconocer y premiar la producción académica escrita en arquitectura, mediante una convocatoria abierta a todas las escuelas de arquitectura de Chile, nace el Concurso Nacional de Reflexión e Investigación Arquitectura Escrita, cuya primera versión se desarrolló a lo largo del año 2017. De esta forma, se destacaron distintas propuestas que permiten discutir sobre las temáticas actuales y diversas formas de pensamiento desarrolladas por estudiantes y recién titulados, entregando un medio para la publicación de sus investigaciones, reflexiones o memorias proyectuales, valorando las ideas desarrolladas en su formación desde la palabra escrita, conociendo cómo se está pensando la arquitectura en las distintas universidades de Chile.

Durante todo el proceso se contó con un total de 30 evaluadores, profesionales y académicos con distintas visiones y provenientes de múltiples campos de acción disciplinares, quienes seleccionaron a los 20 artículos ganadores, menciones y destacados, entre los 87 participantes que representaron a 18 escuelas de arquitectura de 9 ciudades distintas del país, en sus dos categorías: pregrado, para estu- diantes de segundo a cuarto año; y grado, para estudiantes desde quinto año.

La primera versión del concurso fue organizado por Grupo Arquitectura Caliente, con el apoyo de Ediciones ARQ, el auspicio de Color Animal, Materia Arquitectura y Revista de Arquitectura, el patrocinio del Colegio de Arquitectos de Chile y su Comité de Arquitectos Jóvenes y la colaboración de Red de Estudiantes de Arquitectura, AriztíaLAB, Ciudad Atmosférica y Plataforma Arquitectura. Además, se contó con el apoyo de Arte Nostro y Uno a Mil para realizar la presente publicación.

\section{Equipo concurso}

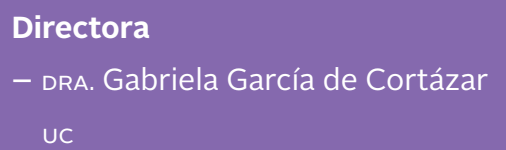

\section{Director curatorial \\ - MG. Francisco Díaz EDICIONES ARQ + UC}

\section{Director ejecutivo}

- ARQ. Sebastián Simonetti GAC

\section{Producción}

Christian Fierro

\section{Asistente de producción}

Constanza Villegas

\section{Comunicaciones}

Consuelo Araneda

\section{Diseño}

Cristina Núñez

\section{Comité de Evaluadores}

- MG. José Abásolo UDLA

- mG. Francisco Allard uRBÁNicA

- MG. Maximiano Atria UCH

- DR. Sergio Baeriswyl uBB

- mg. Tirza Barría UACH

- DR. Rodrigo Booth UCH

- mg. Alejandra Bosch uc

- mG. Eduardo Castillo

- DRA. Alejandra Celedón uc

- mg. Alejandra Cortés uch

- mG. Felipe Corvalán uch

- mG. Renato D'Alençon uc

- mG. Pablo Fuentes PLAZAS DE BOLSILLO

- DRA. Paola Jirón UCH

- ARQ. Layla Jorquera CAJ

- DRA. Natalia Jorquera uch

- MG. Claudio Magrini uDP

- ARQ. Luis Felipe Maureira

REPÚBLICA PORTÁTIL
- MG. Gabriela Medrano unAB

- Mg. Pía Montealegre uDP

- mG. Vesna Obilinovic unAP

- DR. Daniel Opazo uch

- DRA. Amarí Peliowski ARCHIvo VISUAL DE SANTIAGO

- DR. José Rosas uc

- MG. Diego Rossel uch

- mg. Marcelo Ruiz metropolítica

- ARQ. Constanza Sateler DUPLA

- DR. Ricardo Tapia UCH

- mg. Germán Valenzuela utAL

- DRA. Cecilia Wolff ucH 